\title{
Rôle des représentations des apprenants : expérimentation relative au climat motivationnel
}

Linda Breton

\section{CpenEdition}

\section{Journals}

Édition électronique

URL : http://journals.openedition.org/asp/3589

DOI : 10.4000/asp.3589

ISSN : 2108-6354

\section{Éditeur}

Groupe d'étude et de recherche en anglais de spécialité

\section{Édition imprimée}

Date de publication : 1 décembre 1996

Pagination : 307-312

ISSN : 1246-8185

\section{Référence électronique}

Linda Breton, «Rôle des représentations des apprenants : expérimentation relative au climat

motivationnel », ASp [En ligne], 11-14 | 1996, mis en ligne le 11 mai 2013, consulté le 19 avril 2019

URL : http://journals.openedition.org/asp/3589 ; DOI : 10.4000/asp.3589

Ce document a été généré automatiquement le 19 avril 2019

Tous droits réservés 


\title{
Rôle des représentations des apprenants : expérimentation relative au climat motivationnel
}

\author{
Linda Breton
}

\section{Contexte général}

1 Le contexte de l'expérimentation est le suivant. L'Université de technologie de Compiègne (UTC) est à la fois une université et une Grande École, proposant un diplôme d'ingénieur en 5 ans. Les étudiants sont recrutés soit directement après le baccalauréat, soit à baccalauréat +2 .

2 La démonstration d'une certaine compétence dans une langue seconde est obligatoire pour l'obtention du diplôme. Cette compétence est évaluée par certification interne : le niveau minimum est dit «niveau pratique » ou niveau 3. Les niveaux 1 et 2 existent en amont de ce niveau fatidique et les niveaux 4 et 5 après, pour les étudiants capables d'aller au-delà.

3 Nous sommes en train de modifier notre système actuel pour le remplacer par un système d'apprentissage en autonomie guidée.

4 À partir de septembre 1996, les changements suivants se seront produits : une diminution progressive du présentiel; l'ouverture d'un Centre de ressources contenant des «projets » ou valises pédagogiques pour toutes les langues enseignées; un système de suivi individuel par tutorat; des cours d'apprentissage de l'apprentissage (sans doute sur vidéocassette) et le maintien de groupes de 8 à10 pour des activités d'expression.

\section{Contexte particulier}

En tant que responsable du niveau dit 2 en anglais, juste avant le niveau pratique requis pour le diplôme, je m'intéresse particulièrement à certains phénomènes motivationnels. 
Les étudiants qui se trouvent dans cette formation ont fait typiquement entre 6 et 8 ans d'anglais auparavant. Pour des raisons qui ne peuvent pas être purement cognitives, ils ont donc « stagné » ou plafonné à un niveau assez bas dans cette discipline.

Une expérience antérieure que l'on peut désigner grossièrement comme négative, influence inévitablement l'attitude de l'apprenant envers l'apprentissage de l'anglais au moment où il arrive dans la formation dont il est question.

7 C'est dans ce sens d' " attitude » que j'utiliserai le terme "représentation », tout en étant consciente que ce même terme recouvre d'autres notions plus ou moins voisines. Une représentation, pour notre propos, est donc un construit complexe, dépendant à la fois de l'expérience antérieure, de facteurs affectifs (personnalité, etc.) mais aussi de la nature du problème à résoudre. À mon sens, un apprenant à une représentation: de sa compétence, de l'efficacité de ses efforts et du but qu'il se fixe

Il est clair que ces représentations affectent la motivation de l'apprenant et sont donc une variable psychologique et pédagogique importante. L'expérimentation que nous envisageons pour le semestre qui débute en septembre part de l'hypothèse qu'il est possible d'intervenir au niveau de cette variable.

9 Nous allons maintenant essayer d'éclairer ce phénomène un peu mystérieux qui n'est évidemment pas observable directement.

\section{L'apprentissage comme comportement d'accomplissement}

10 Cette approche est développée surtout en psychologie du sport (Famose 1991) mais il y a en fait beaucoup d'idées en commun entre la didactique des langues et la didactique du sport.

11 Si l'on accepte la vision cognitiviste qui voit toutes nos actions comme orientées vers un but (goal-directed) (Anderson 1985), en ce qui concerne l'apprentissage le but est l'accomplissement. Or, il y a deux façons de s'accomplir: vis-à-vis des autres ou d'une norme externe et vis-à-vis de soi-même. Dans le premier cas, la motivation est compétitive ; dans le deuxième, c'est une motivation « de maîtrise ».

12 L'idée de se mesurer aux autres ne convient pas nécessairement à tout le monde, tant s'en faut. Chacun de nous a connu des cas où un climat compétitif s'est même avéré contreproductif. Mais indépendamment de toute considération psychologique, il se trouve que le remplacement de groupes par le travail autonome annule en partie le contexte dans lequel cette sorte de motivation peut opérer. ( $\mathrm{Si}$, toutefois, dans le système d'autonomie guidée, on retient une phase d'évaluation normative, la motivation compétitive subsiste.)

13 De toute façon, le contexte d'un système d'apprentissage en autonomie guidée semble exiger une attention particulière à la motivation de l'apprenant seul avec lui-même et sa tâche. La théorie de l'Attribution causale (Weiner 1974 et 1985) peut fournir des pistes intéressantes pour étudier l'apprentissage en tant que comportement d'accomplissement.

\section{La théorie de l'Attribution causale}

14 Selon la théorie de l'Attribution causale (Attribution Theory), l'homme est ainsi fait qu'il cherche toujours à expliquer les résultats de ses actions. Plus précisément, chaque fois 
qu'il réalise une action aboutissant à un résultat, il ressent un besoin de trouver une explication pour ce résultat, de lui attribuer une ou plusieurs causes.

\section{Nature des tâches}

Grosso modo, on distingue quatre types de causes dans une situation de comportement d'accomplissement : la compétence, l'effort, la difficulté de la tâche et le hasard.

Psychologiquement, ces causes correspondent à trois dimensions : la dimension interneexterne, stable-instable et contrôlable-incontrôlable.

Au premier niveau, la compétence et l'effort sont considérés comme des causes internes, tandis que la difficulté de la tâche et le hasard sont externes. Au deuxième niveau, la compétence et la difficulté de la tâche sont stables, alors que l'effort et le hasard sont instables. Au troisième niveau, l'intensité de l'effort est contrôlable, mais la difficulté objective de la tâche ne l'est pas.

Il me semble que le double apport de la notion de l'apprentissage comme comportement d'accomplissement et la théorie de l'Attribution causale nous aide à mieux comprendre les représentations des apprenants dont il est question.

Étant donné que chacun cherche à multiplier les réussites et à éviter les échecs, la situation la plus valorisante est celle où une réussite est attribuable à des causes internes, stables et contrôlables. À l'inverse, la situation la moins valorisante est celle où un échec est à imputer à ces mêmes causes.

Il se peut qu'une absence de réussite dans le domaine de l'apprentissage de l'anglais ait négativement influencé les représentations qu'ont les apprenants (du niveau 2) de leur compétence, de l'efficacité de leurs efforts, voire de la nature du problème à résoudre.

\section{L'expérimentation au niveau des représentations}

Nous envisageons d'intervenir à deux niveaux : 1) par l'intermédiaire de la nature des tâches proposées ; 2) par l'intermédiaire de l'organisation du système d'apprentissage.

3 Les «ensembles de tâches" (ou projets) qui seront disponibles dans le Centre de ressources sont conçus selon un modèle qui a comme ambition de reconstituer les étapes $\mathrm{du}$ processus mental d'acquisition. Nous distinguons quatre phases, à ne pas confondre avec les niveaux internes 1 à 4 (cf. tableau 1). 


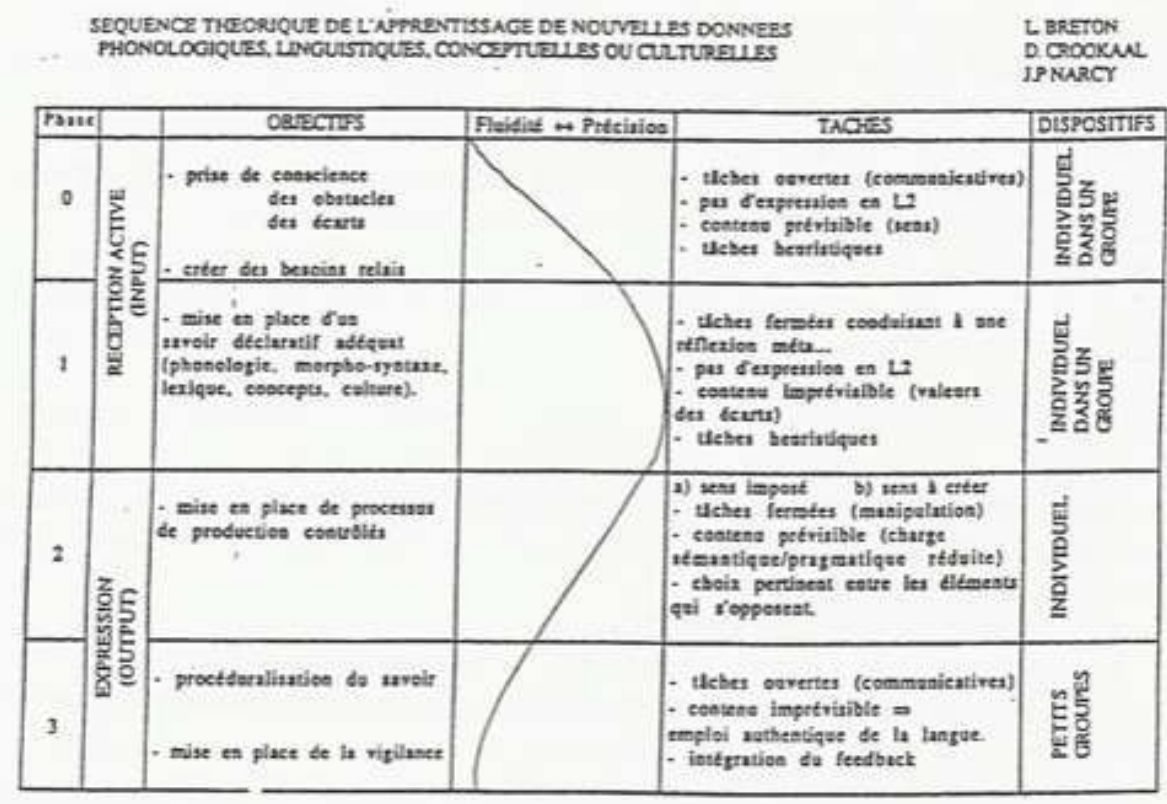

d'après Anderson (1985)

Il pourra choisir librement un nombre fixe (disons 5) de projets parmi la gamme disponible à son niveau. Il aura un binôme pour le travail d'expression (activités à écart d'information essentiellement) et sera affecté à un petit groupe qui se réunira pendant une heure par semaine, pour des activités communicatives.

Cette grille a été inspirée en partie par l'analyse faite par Anderson (1985) concernant le développement de « l'expertise », analyse reprise par O'Malley et Chamot (1990).

Dans la colonne « dispositifs », nous avons fait la différence entre les phases qui sont à effectuer individuellement et celles qui ne peuvent s'effectuer convenablement sans interlocuteur. Les ensembles de tâches, ou projets sont très structurés, de sorte que l'apprenant a une idée de la faisabilité du tout dès le début. Chaque «sous-tâche » est bien délimitée dans le souci de rentabiliser le temps de nos étudiants, que nous savons précieux. Enfin, chaque projet porte une description de son contenu, des points de grammaire qui y sont traités, etc. On pourrait parler d'une « table des matières ».

\section{L'organisation du système}

L'apprenant sera encadré grâce à un suivi individuel par tutorat (un $1 / 4 \mathrm{~h}$. par semaine). Au fur et à mesure que les projets seront menés à bien, ils seront évalués par le tuteur, avec la participation de l'étudiant (qui évalue à la fois ses résultats, son parcours et la tâche elle-même). 


\section{Effets souhaités sur les représentations}

Grâce à cette double approche, nous pensons influencer favorablement les représentations des apprenants, de la manière décrite ci-dessous.

Représentation de sa compétence (rappel: la compétence est une cause interne et stable)

- absence de climat compétitif, climat de motivation de maîtrise permet une meilleure focalisation sur la tâche

- l'apprenant mesure son progrès par rapport à lui-même

- chaque projet complété le rassure s'il a été réussi ou le renseigne pour le prochain s'il a été moins réussi.

Représentation de l'efficacité de ses efforts (rappel : l'effort est une cause interne et contrôlable)

- l'organisation générale du système (peu de présentiel) vise la rentabilisation du temps (précieux) de l'étudiant

- la division du but à atteindre en sous-buts (les 5 projets) doit réduire l'effet de monotonie et la sensation d'essoufflement qui a tendance à remplacer l'enthousiasme initial, permettant le maintien de l'effort.

Représentation de la difficulté de la tâche (rappel: la difficulté de la tâche est une cause externe, stable et incontrôlable)

- la «table des matières » du projet permet à l'apprenant d'avoir une vision globale des différentes étapes et une idée de la faisabilité de l'ensemble

- la nature structurée du projet lui donne le sentiment d'être encadré

- l'étudiant peut progresser à son rythme et passer plus de temps sur une partie ou une autre, s'il en a besoin

- ces causes deviennent moins externes et contrôlables.

Représentation du hasard (rappel: le hasard est une cause externe, instable et contrôlable)

- le fait d'avoir un choix, même limité, de projets éloigne le sentiment de hasard pur ou d'un sujet imposé par un autre

- le fait de laisser une place à l'auto-évaluation des projets réduit le caractère aléatoire d'une évaluation entièrement hétérogène

- le hasard est partiellement internalisé et contrôlé.

Les effets escomptés seront difficilement mesurables, surtout à court terme, mais nous pourrons peut-être vous en parler dans un an.

\section{BIBLIOGRAPHIE}

Anderson, J. R. 1985. Cognitive Psychology and its Implications. New-York : W.H. Freeman.

Famose, J-P., P. Fleurance et Y. Touchard.1991. L'Apprentissage moteur. Paris : Éditions Revue EPS. 
Narcy, J.-P. Apprendre une langue étrangère. Paris : Les Éditions d'Organisation.

O’Malley, J. M. et A. U. Chamot.1990. Learning Strategies in Second Language Learning. Cambridge : Cambridge University Press.

Weiner, B. 1974. Achievement Motivation as Attribution Theory. Morristown : General Learning Press.

Weiner, B. 1985. « An attribution theory of achievement motivation ». Psychological Review 4, 548-573.

\section{RÉSUMÉS}

En ce qui concerne l'apprentissage de l'anglais, une partie du public des étudiants ingénieurs voit sa progression freinée par une mauvaise représentation de sa compétence, de l'efficacité de ses efforts et du but à atteindre. La théorie de l'Attribution causale fournit des pistes pour étudier l'apprentissage en tant que comportement d'accomplissement, dans le but d'agir favorablement sur cette représentation, qui est une variable psychologique importante. Il est suggéré qu'un apprentissage en autonomie-guidée spécifiquement conçu pourra mieux convenir à cette catégorie d'étudiant.

As far as learning English is concerned, the beliefs and expectations of one category of engineering student regarding his competence, the efficacy of his efforts and his goal, are an obstacle to his progression. Attribution Theory can provide insights for the study of the language learning process as goal-directed behaviour, with a view to influencing favourably these beliefs and expectations, which constitute an important psychological variable. It is suggested that a specially designed guided autonomy course would be better suited to this category of student.

\section{INDEX}

Mots-clés : attribution causale, autonomie (guidée), motivation, représentation

Keywords : attribution theory, autonomy (guided), belief, motivation

\section{AUTEUR}

\section{LINDA BRETON}

Linda Breton est enseignant-chercheur à l'Université de technologie de Compiègne (UTC) depuis huit ans, où elle est responsable du niveau intermédiaire en anglais et d'une UV sur l'Union européenne enseignée en anglais. Elle prépare actuellement une thèse, sous la direction de JeanPaul Narcy, sur les options méthodologiques et opérationnelles d'un système d'apprentissage de l'anglais en autonomie guidée. Elle a fait deux communications pour l'UPLEGESS (Colloque Nantes 1995 et Congrès Compiègne 1996) et une communication au 3rd International Conference of the Association for Language Awareness à Dublin en juillet 1996. 